\title{
DIE KURISCHEN RHOMBISCHEN LANZENSPITZEN MIT DAMASZIERTEM BLATT *
}

\author{
VON \\ A. ANTEINS
}

BEI der Forschung der auf dem Territorium der Baltischen Sowjetrepubliken gefundenen Lanzenspitzen mit damasziertem Blatt konnte man eine Gruppe von 65 Exemplaren aussondern, die sich durch ein rhombisches Blatt mit annähernd quadratischem Gipfel von den anderen unterscheiden. Da die Funde hauptsächlich aus dem von den alten Kuren bewohnten Gebiet stammen (40 ex.; Abb. 1, Nr. 6-18 und Nr. 30-33), werden sie auch oft als kurische Lanzenspitzen genannt. Diese Lanzenspitzen stammen aus den XI.-XII. Jh. ${ }^{8}$, aber - davon zeugen die Funde in Durbe - wurden sie auch im XIII. Jh. gebraucht ${ }^{10}$. Die Länge dieser Lanzenspitzen erreicht $390 \mathrm{~mm}$ (Abb. 2).

Von der Gesamtzahl der oben erwähnten kurischen Lanzenspitzen mit damasziertem Blatt befinden sich $32 \mathrm{Ex}$. in dem historischen Museum der Lettischen SSR (weiter durch LVM bezeichnet), 12 Ex. in dem Liepaja Museum für Historie und Kunst (LM), 8 Ex. in der archäologischen Abteilung des Hist. Instituts der Akademie der Wissenschaften der Estnischer SSR (IZA), 5 Ex. im Historisch-ethnographischen Museum zu Vilnius, Litauen (VM), 2 Ex. im Landesmuseum zu Paide, Estland (PM), und je ein Ex. in Leningrad - in der Eremitage (LE), in der III Abteilung des Moskauer staatlichen Museums (GIM), in der archäologischen Abteilung des Hist. Instituts der Akademie der Wissenschaften der Lettischen SSR (LZA), im Landesmuseum zu Madona, Lettland (MM), im Historischen Stadtmuseum zu Kaunas (KM), und im Landesmuseum zu Ukmerge, Litauen (UM).

Nach dem Ornament des Blattes lassen sich die genannten Lanzenspitzen in 7 Gruppen einteilen, 'was ungefähr die Hälfte von den im Baltikum festgestellten 13 Gruppen ausmacht ${ }^{3}$. Ihre zahlenmässige Aufteilung geht aus der Tabelle 1 hervor, in der auch die Zahl der Analysen der Mikrostruktur 'wie auch der Phosphormenge angegeben sind (p. 25). Alle Lanzenspitzen, die einer Mikroanalyse unterworfen wurden, hatten eine teilweise oder völlig ausgeglühte Metallstruktur. Dies zeugt davon, dass die Lanzenspitzen einst dem Ritual der Feuerbestattung ausim Text.

* Die Nummern des Literaturverzeichnisses entsprechen den Anmerkungsziffern 
gesetzt worden sind. Dieser Umstand verhinderte die Struktur der Lanzenspitzen in der Zeit ihrer Verwendung festzustellen.

Die Methoden der Makro- und Mikroanalysen sind in unsren vorigen

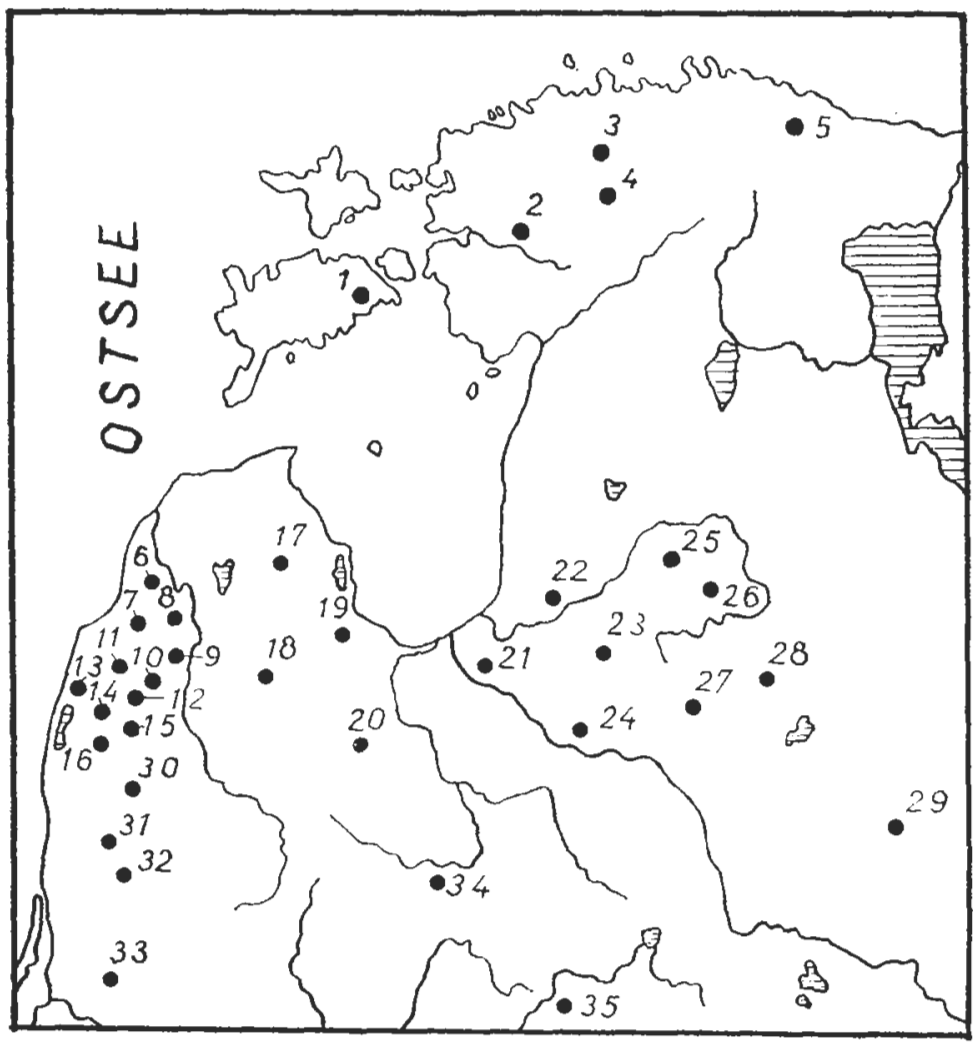

Aвв. 1.-Fundorle der kurischen rhombischen Lanzenspitzen mit damaszierlem Blall: a) Esiniscbe SSR: 1. Randvere; 2. Haimre; 3. Tarlia; 4. Paide; 5. Haljala; Estland (1 Ex.). b) Lettische SSR; 6. Pasilciems; 7. Alsunga (2 Ex.); 8. Iwande (2 Ex.); 9. Kapenieki (2 Ex.); 10. Kasdanga; 11. Aispule; 12. Stroki; 13. Medse (2 Ex.); 14. Durbe (11 Ex.); 15. Bunkas; 16. Kaleti; 17. See Wilkmuischa (4 Ex.); 18. Saldus; 19. Tukums; 20. Bukaiscbi; 21. Salaspils; 22. Turaida; 23. Allascbi (4 Ex.); 24. Aiskraukle (4 Ex.); 25. Rauna; 26. Piebalga; 27. Kalsnawa; 28. Zeswaine; 29. Scbaureiti; Kurzeme (5 Ex.). c) Lilauiscbe SSR; 30. Apuola; 31. Laiviai; 32. Nausodis (2 Ex.); 33. Scbwekscbnit; 34. Linksmulschiai; 35. Ukmerge. 


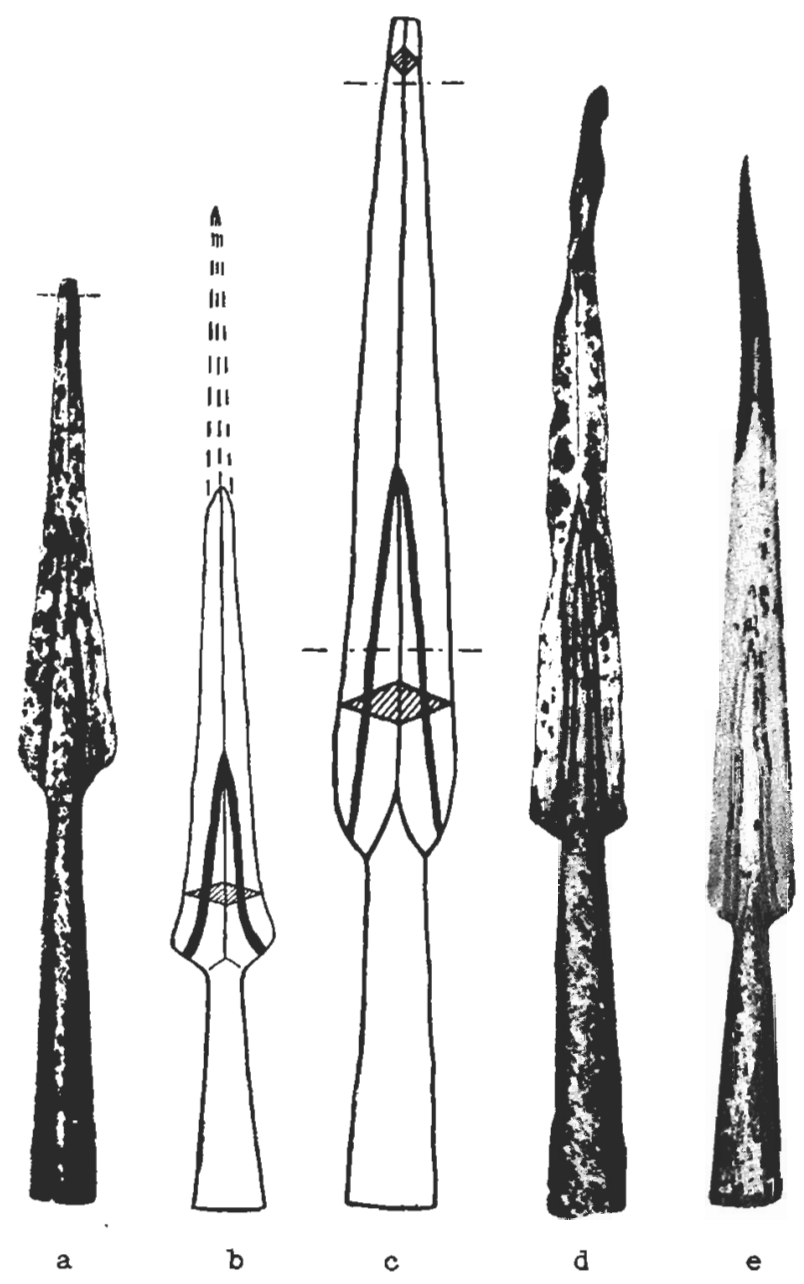

Aвв. 2.-Die Lanzenspitzen mit scbmalen Streifen: a) Kapenieki (1/3); b) Durbe (LM 13212; 1/3); c) Kaleti (1/2); d) Kurzeme (LVM 64953; 1/3); e) Stroki (1/3).-Die Stelle des Querscbnitts. 


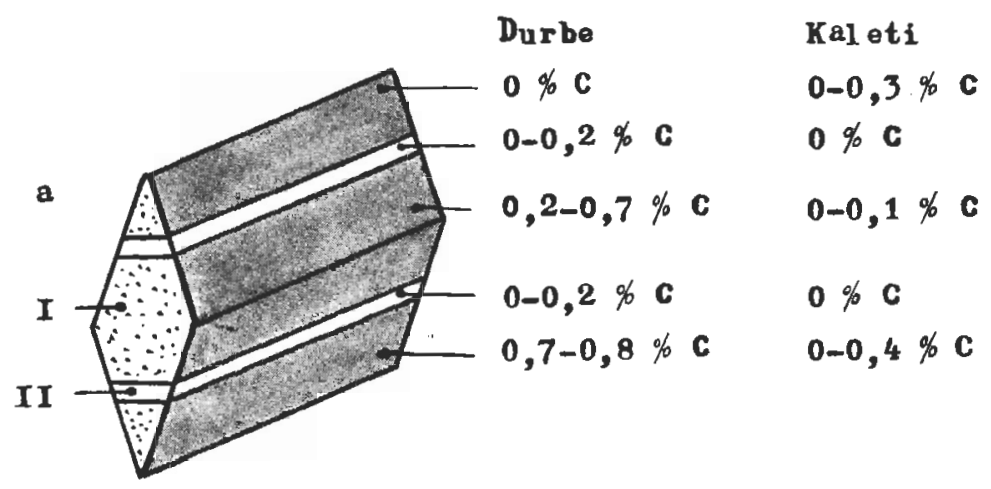

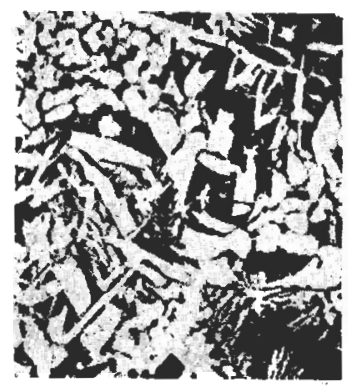

b

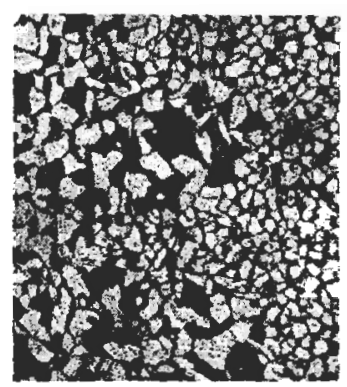

d

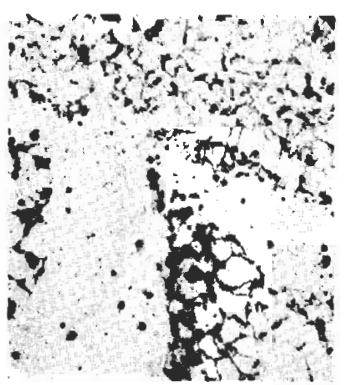

c

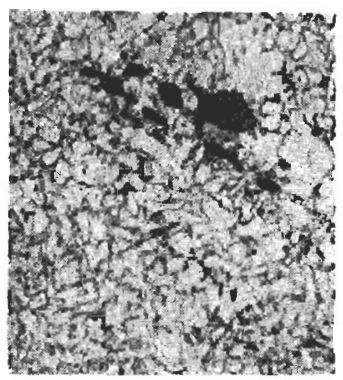

e

Aвв. 3.--Technologisches Schema des Blatles, welches beiderseits vom Mitlelgrat cinen schmalen Streifen hal(a) und die Mikrostruktur des Blattes (Vergr. 100 X): b-c) Durbe; d) Kaleti; e) Kapenicki. 
Arbeiten beschrieben 1, 2.6. Zur Erforschung der Ungleichmässigkeit des Phosphors wurde das Rezept von H. Klemm verwendet ${ }^{7}$.

\section{Die Lanzenspitzen mil Blall, das beiderseits vom Mitlelgrat einen schmalen oder zwei schmalen Streifen bat}

Solche Lanzenspitzen sind durch 23 Ex. belegt; 15 Ex. haben beiderseits des Mittelgrats einen Streifen, und 8 Ex. haben zwei Streifen. Lanzenspitzen mit einem Streifen sind gefunden in: Alsunga (LVM-V 4157), Kapenieki (LVM-V 7645:1), Medse (LVM-A 10789:65), Durbe (LM-12984; 13212), Bunkas (LVM-V 8571:46), Kaleti (LM-13952), Allaschi (IZA-1954:2), Aiskraukle (LVM-13162), Zeswaine (MM-663), Apuole (VM-V 8511:5), Laiviai (VM-Em2:128), Nausodis (VM-AR 7:10; AR 7:11), Kurseme (LVM-30A:90). Lanzenspitzen mit zwei Streifen in: Durbe (LVM-V 1783; LM-12945), Iwande (LVM-V 383), Stroki (LVMII 2056), Kurseme (LVM-64953), Schwekschnji (KM-835:22), Allaschi (IZA-1958:1), Aiskraukle (LVM-I 3153). Drei Lanzenspitzen mit einem Streifen und zwei mit zivei Streifen sind auf der Abb. 2 zu sehen (Abb. 2).

Die kürzeste von den nicht abgebrochenen Lanzenspitzen dieser Gruppe ist die in Apuole gefundene (193 mm lang), die längste ist eine in Kutzeme gefundene zweistreifige Lanzenspitze ( $350 \mathrm{~mm}$ lang). Die Länge der Tülle variiert in breiten Grenzen: die kürzeste Tülle etwa $60 \mathrm{~mm}$ (aus Laiviai) und die längste Tülle etwa $170 \mathrm{~mm}$ (aus Aiskraukle, LVM-13262).

Um das technologische Schema aufzustellen und die Struktur zu erforschen, wurden Querschnitte von fünf Lanzenspitzen (zwei aus Durbe, LM-12984; 12945, eine aus Kaleti, eine aus Kapenieki und eine aus Aiskraukle, LVM-I 3153) gemacht. Das technologische Schema und die Mikrostruktur eines Blattes, welches beiderseits vom Mittelgrat einen schmalen Streifen hat, sind auf der Abb. 3 wiedergegeben. Technologisches Schema und Mikrostruktur eines Blattes mit zwei schmalen Streifen auf den beiden Seiten des Mittelgrats findet man auf Abb. 4.

Das technologische Schema des Blattes mit einem Streifen (Abb. 3-a) aus Durbe und Kaleti zeigt weiter den Kohlenstoffgehalt in jedem Teil des Blattes. Es lässt sich feststellen, dass die Blätter nicht aus demselben Metall verfertigt worden sind. Daraus ergibt sich, dass die Lanzenspitzen von verschiedenen Waffenschmieden hergestellt wurden. Der Mittelteil des Blattes aus Durbe ist aus Stahl mit ungleichmässigem Kohlenstoffgehalt $(0,2-0,7 \%)$ - die Mikrostruktur der Stelle $\mathrm{I}$ ist auf der Abb. 3-b dargestellt, an der Ferrit und Perlit zu sehen sind; die des Blattes aus Kaleti - dagegen aus Eisen, welches an einigen Stellen bis $0,1 \%$ Kohlen- 
stoffgehalt aufweist. Die Streifen des Blattes von Durbe wollte man wohl aus einem gewundenen, gedrehenen Eisen-Stahl Streifen angefertigt haben, wie es von der Abb. 9, bei den unter $b$ und $c$ gezeigten Lanzenspitzen hervorgeht. Dies ist aber dem Verarbeiter nicht gelungen. An

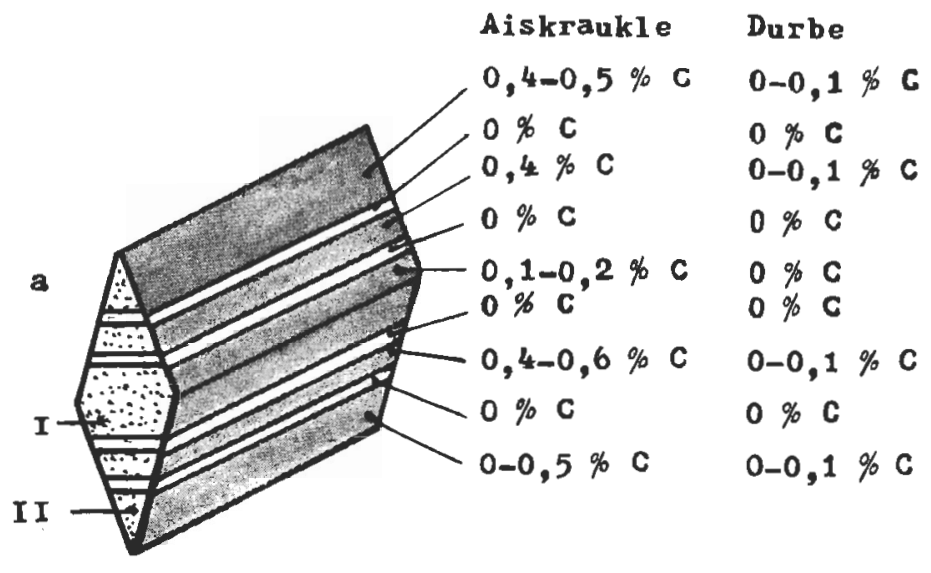

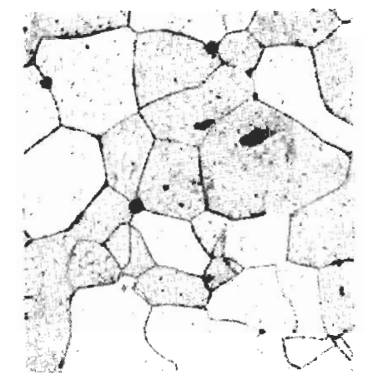

b

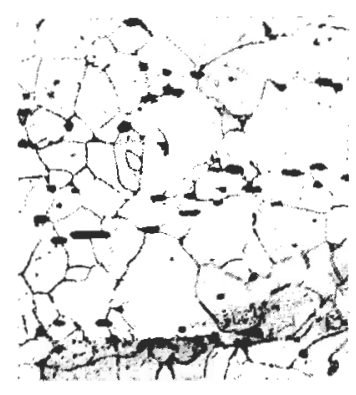

c

Авв. 4,-Technologisches Schema des Blattes, welches beiderseils vom Mittelgrat zwei schmale Streiten hat(a) und die Mikrostruktur des Blattes (Vergr. $100 X)$ aus Durbe ( $b$ und $c$ ).

derer Stelle sehen wir einen Streifen aus Eisen (Ferrit) mit Fragmenten von Stahllamellen an einzelnen Stellen. Die Mikrostruktur dieser Fragmente (der Stelle II) ist auf der Abb. 3-c gezeigt, auf welcher im hellen 
Ferritfelde die Fasern des Ferrit und Perlit der Stahllamellen zu sehen sind (die Lamellen enthalten etwa 0,2\% Kohlenstoff).

Die Lanzenspitzen aus Kaleti haben, wie auch viele anderen Blätter, die Streifen aus kohlenstofflosem Ferrit. Dieser Ferrit aber ist grobkörnig und weist beim Ätzen die Grenzen seiner Körner schlecht auf. Sein Aussehen ist ähnlich der auf der Abb. 10-c gezeigten Struktur. Der Ferrit des Streifens enthält eine bedeutendere Menge von Phosphor. Dic beiden Schneidenspitzen der Lanzenspitzen aus Durbe sind nicht von gleicher Qualität. Während eine Schneidenspitze aus Stahl mit Kohlenstoffgehalt 0,7-0,8\% geschmiedet ist, so ist die andere aus Eisen. Die erste Schneidenspitze erhält beim Härten gute Schneide-Eigenschaften. (Aus Stahl mit Kohlenstoffgehalt 0,8-0,9\% sind auch die Schneidenspitzen der besten Lanzenspitzen schon im IX. Jh. verfertigt worden, $z . B$. der in Salaspils gefundenen mit silberplattierter Tülle, und andere ${ }^{2,3}$.) Die Schneide-Eigenschaften der zweiten sind indessen schlecht, denn sie lässt sich nicht härten und wird deswegen viel zu weich.

Die beiden Schneidenspitzen von diesen Lanzenspitzen aus Kaleti sind von det gleichen Qualität. Sie erhalten Kohlenstoff bis 0,3-0,4\% und beim Härten werden sie verhältnismässig hart. Dasselbe Metall ist auch im Gipfel. Die Mikrostruktur des Querschnitts durch den Gipfel der Lanzenspitzen aus Kaleti ist auf der Abb. 3-d zu sehen, wo man Ferrit und Perlit (Kohlenstoffgehalt etwa $0,3 \%$ ) erblicken kann. Es ist zu bemerken, dass in diesem Querschnitt auch solche Mikrostellen zu sehen sind, 'wo nur Ferrit sichtbar wird.

Wie die Analyse des Querschnitts durch den Gipfel der Lanzenspitze aus Kapenieki zeugt, ist diese aus einem anderen Metall als die oben er'wähnten, geschmiedet worden. Die Mikrostruktur des Querschnitts (Abb. 3-e) besteht aus Ferrit, der etwas feinen Perlit und Zementit enthält (C-Gehalt bis $0,1 \%$ ).

Bei der Betrachtung des Kohlenstoffgehalts der Lanzenspitzen mit zwei Streifen aus Aiskraukle und Durbe, geht es aus dem technologischen Schema (Abb. 4-a) hervor, dass diese Blätter, ebenso 'vie die einstreifigen, nicht aus demselben Metall verfertigt worden sind. Ausgenommen sind die Streifen selbst. Von der Qualität der Schneidenspitzen ausgehend, ist die Lanzenspitze aus Durbe qualitätsmässig geringer als die aus Aiskraukle, denn die Schneidenspitzen des Blattes der ersten sind aus Eisen mit C-Gehalt bis $0,1 \%$, während von der anderen eine Schneidenspitze $0,4-0,5 \%$ und die andere bis $0,4 \%$ Kohlenstoff enthält, 'was ihnen beim Stählen eine bedeutende Härte verleiht.

Das Metall der Streifen in den beiden Lanzenspitzen ist dasselbe: seine Mikrostruktur besteht aus grobem Ferrit und ist der des einstreifi- 
gen Blattes auf der Abb. 10-c gezeichten Mikrostruktur ähnlich. Das Metall der Streifen enthält eine bedeutende Phosphormenge.

Die Mikrostruktur in der Mitte des Blattes (die Stelle I) der Lanzenspitze aus Durbe ist auf der Abb. 4-b zu sehen: man sieht Ferrit mit einigen feinen Schlackenbeisätzen. Die Mikrostruktur der Schneidenspitze (die Stelle II) sieht man auf der Abb. 4-c. Da sieht man auch Ferritkörner mit einigen feinen Zementitkörnchen und Einschliessungen von gedehnten Schlacken.

Vergleichen wir das Ornament dieser Gruppe mit den auf der Abb. 9 gezeigten Ornamenten der Lanzenspitzen, muss man gestehen, dass es sehr schwer fällt, diese Lanzenspitzen wegen des einfachen Streifenornaments als damaszierte zu bezeichnen. Deswegen 'werden die Erzeugnisse dieser Art nicht damaszierte, sondern ornamentierte genannt, und den dazu verwendeten Stahl bezeichnet man als Ornamentenstahl. Um aber die Kunst der Herstellung des auf der Abb. 9 gezeigten, damaszierten Stahls zu erlernen, hatte man von der einfachsten, leichtesten Art des Schmiedeschweiss-ornamentes ausgehen müssen. Und so einen haben die Lanzenspitzen dieser Gruppe. Deswegen hätte man den Stahl der Blätter dieser Gruppe als einen auf die einfachste (primitiveste) Weise damaszierten Stahl benennen können.

Mit Rückblick auf die Form kann man diese Gruppe von 23 Lanzenspitzen noch in Untergruppen einteilen. So ist z.B. die Lanzenspitze aus Kapenieki (Abb. 2-a) der einstreifigen aus Aiskraukle ähnlich; die Lanzenspitze aus Durbe (b) ist der aus Alsunga ähnlich; die Lanzenspitzen aus Kaleti (c) sind den beiden einstreifigen aus Nausodie, wie auch den aus Zeswaine und aus Medse ähnlich; die Lanzenspitze aus Stroki (e) hat Ähnlichkeit mit fünf zweistreifigen (aus Iwande, Durbe [LM-12945], Schwekschnji, Aiskraukle und Allaschi). Die einstreifigen Lanzenspitzen aus Laiviai, Apuole, Kurseme sowie eine aus Durbe (LM-12984) bilden mit ihren kurzen Tüllen (die Länge der Tülle $=60-70 \mathrm{~mm}$ ) eine andere Untergruppe.

\section{Die Lanzenspitzen deren Blatt beiderseits vom Mittelgrat eine Zwiscbenlage mit gezacktem Rand oder eine Zwischenlage mit gezacktem Rand und einen schmalen Streifen hat}

Hierher gehören 37 Lanzenspitzen. Von ihnen haben 6 Ex. ein Blatt mit nur einer Zwischenlage mit gezacktem Rand, 'während $31 \mathrm{Ex}$. eine Zwischenlage mit gezacktem Rand und einen schmalen Streifen haben. 


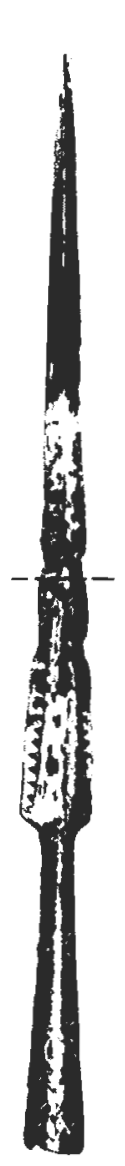

a

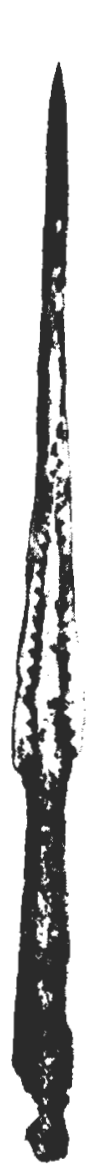

b

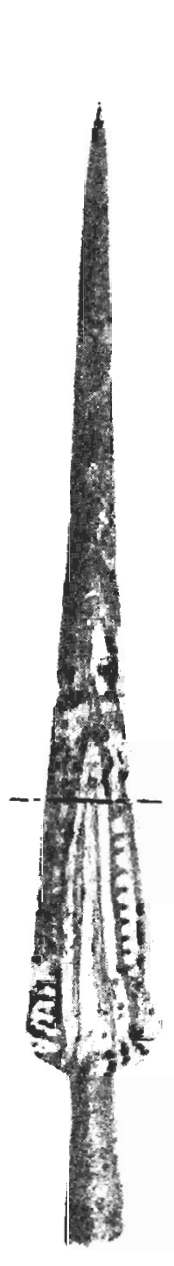

c

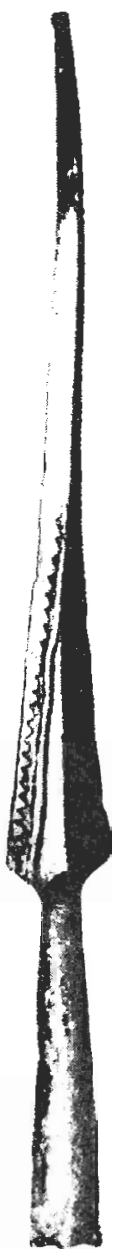

d

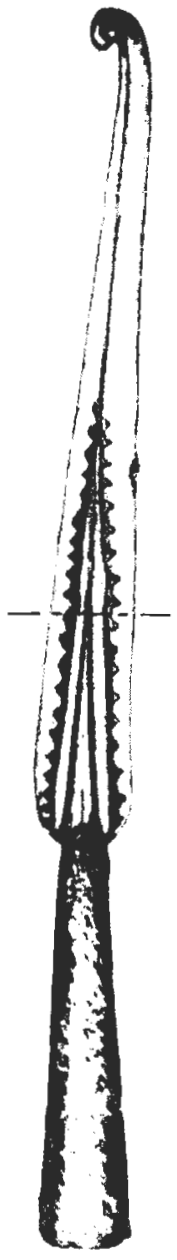

A

fil

III

III

ll

111

111

111

111

111
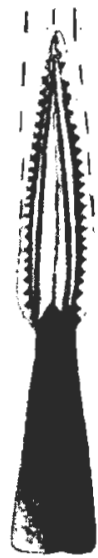

$f$

Aвв. 5.-Die Lanzenspitzen mit einer Zwiscbenlage mit gezacktem Rand oder mit einer Zwischenlage mit gezacktem Rand und scbmalen Streifen: a) Alsunga (1/3); b) See Wilkmuischa (1/3); c) Durbe (LM 13141; 3/8); d) Iwande (1/3); e) Kasdanga (1/2); f) Linksmutscbiai (1/3).-Die Stelle des Querschnitts. 
Von den ersten sind zwei auf der Abb. 5-a und b, von den zweiten sind vier auf der Abb. 5-cf, zu sehen.

Blätter (Lanzenspitzen), die eine Zwischenlage mit gezacktem Rand haben, sind in: Alsunga (LVM-V 9562:15), Durbe (LM 13192), Kapenieki (LVM-V 7628:3), See Wilkmuischas (LVM-V 8170:91), Turaida (LVM-I 473') und in Haimre (IZA 2727:230) gefunden 'worden.

Blätter mit einer Zwischenlage mit gezacktem Rand und einem schmalen Streifen kamen an den folgenden Fundstätten vor: Durbe (LM 13141; 13219; 13230; 13157), Kasdanga (LVM-V 7651:1), Kurseme (LVM-II 2045), Iwande (LVM-V 385), Medse (LVM-Kompl. 77), Pacilciems (LE 890/929), Aiskraukle (LVM-PV 24618; I 3108), Allaschi (IZA 1959:1; 1954:1), Kalsnawa (LVM-V 1048), Estland (IZA 3884?), Haljala (IZA 83:222), Paide (PM 70/A71:2), Randvere (IZA 3895:388), Bukaischi (LVM-A 10699:7), Tukums (LVM-V 8369:9), Linksmutschiai (VM-AR 31:79), Aispute (LVM-V 7833:27), Kurseme (LVM 30A/69; II 2048), Piebalga (LZA [-]), Rauna (LVM-TL XXXVI), Salaspils (LVM-VVM 2386), Schaureiti (GIM 80135-1235 Nr. 1), See Wilkmuischas (LVM 1763; V 8170:93; V 8170:94). Siehe Abb. 5.

Von den nicht abgebrochenen Lanzenspitzen dieser beiden Gruppen ist das am kürzesten Exemplar $(\mathrm{L}=265 \mathrm{~mm})$ das aus Bukaischi, das längste $(\mathrm{L}=390 \mathrm{~mm})$ das aus I"wande. Die Länge der Tülle schwankt von $65 \mathrm{~mm}$ (Linksmutschiai) bis $115 \mathrm{~mm}$ (Medse).

Um das technologische Schema aufzustellen und die Mikrostruktur zu erforschen, 'wurden Querschnitte von vier Lanzenspitzen gemacht (Alsunga, Durbe [LM 13141], Kasdanga und Aiskraukle [LVM-I 3108]). Das technologische Schema und die Mikrostruktur des Blattes mit einer Zwischenlage mit gezacktem Rand (aus Alsunga) ist auf Abb. 6 gezeicht, und von einem Blatte mit einer Zwischenlage mit gezacktem Rand und einem schmalen Streifen auf Abb. 7 gezeicht (Abb. 6, 7 und 8).

Das Metall des Blattes der Lanzenspitze aus Alsunga hat grosse Ähnlichkeit mit dem Mctall des oben beschriebenen aus Kaleti (siehe das technologische Schema Abb. 3-a und Abb. 6-a). Der Unterschied zwischen diesen beiden Blättern liegt lediglich im Ornament: das erste Blatt hat anstatt des Streifens eine Zwischenlage mit gezacktem Rand. Die Mikrostruktur der Stelle I (Abb. 6-a) der Lanzenspitze aus Alsunga ist auf der Abb. 6-b gezeigt: an der Oberfläche ist Ferrit des Mittelteiles - am Unterteil - Ferrit der Zwischenlage mit gezacktem Rand und Schlackeneinschliessungen zu sehen. Die Mikrostruktur der Stelle II derselben Lanzenspitze ist auf Abb. 6-c zu sehen: am Rand der Oberfläche ist der Ferrit der Zwischenlage mit gezacktem Rand - im übrigen Teil - Ferrit und Perlit der Schneidenspitze zu sehen. 
Die Ergebnisse der Analysen von Querschnitten aus den übrigen drei Lanzenspitzen beweisen, dass ihre Schneidenspitzen mit geringer Schneidefähigkeit sind, 'weil sie für das Härten den benötigten Kohlenstoffgehalt nicht enthalten: das Metall der Schneidenspitzen der Lanze aus Aiskraukle enthält bis $0,2 \%$ Kohlenstoff, die der Lanzenspitze aus Kasdanga und Durbe sind aus Eisen (Abb. 7-a; die Mikrostruktur der Stelle II der

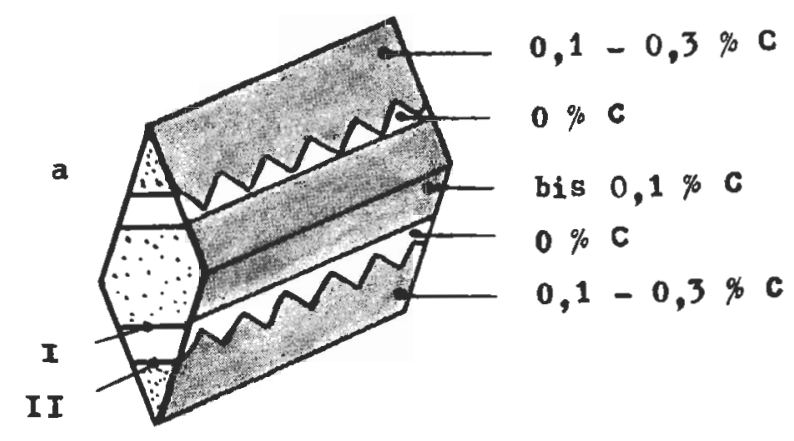

Авв. 6.-Tecbnologisches Schema des Blattes, welches beider. seits vom Mittclgrat eine Zwischenlage mit gezacktem Rand bal(a) und die $M i$ krostruktur (Vergr. $100 \mathrm{X})$ des AlsungaBlaites ( $b$ und $c$ ).

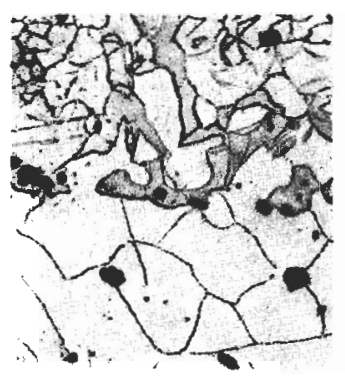

b

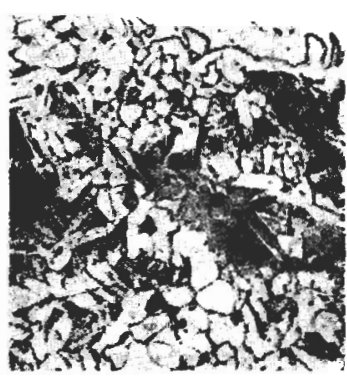

c

Lanzenspitze aus Kasdanga ist auf der Abb. 7-c - die der aus Durbe auf Abb. 7-e zu sehen; auf beiden Abbildungen ist Ferrit zu sehen). Die Mikrostruktur der Stelle I der Lanzenspitze aus Kasdanga ist auf der Abb. 7-b gezeigt und die der Lanzenspitze aus Durbe - auf der Abb. 7-d. Aus den beiden letzten Abbildungen geht es hervor, dass die Blätter beider Lanzenspitzen in diesem Teil aus Ferrit mit einer kleinen Beimischung von Perlit bestehen. 


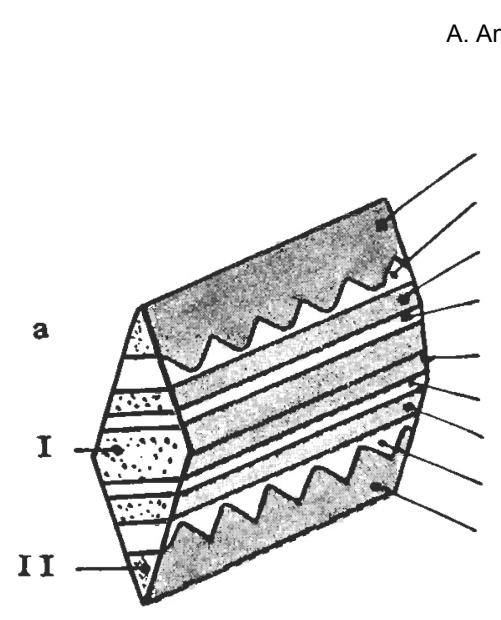

A. Anteins

Aiskraukle

$0-0,2 \%$ c $\quad$ o $\%$ C

0 \% C

$0-0,1 \% \mathrm{C}$

$0 \% \mathbf{C}$

$0,2-0,5 \% \mathrm{c}$

$0 \% \mathbf{C}$

$0,1-0,2 \% c$

$0 \% \mathbf{c}$

$0-0,2 \% \mathrm{C}$

\section{Durbe}

$0 \% \mathbf{c}$

$\mathbf{0} \% \mathbf{C}$

$0 \% \mathrm{C}$

$0 \% \mathbf{C}$

$\mathbf{0} \% \mathbf{C}$

$0 \% \mathbf{c}$

o\% C
Gladius, VII (1968), pp. 5-26 ISSN 0435-029X

$0,1-0,2 \% \mathrm{C}$

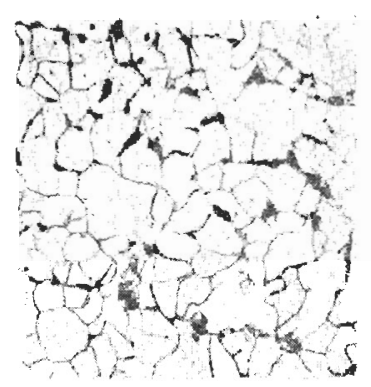

b

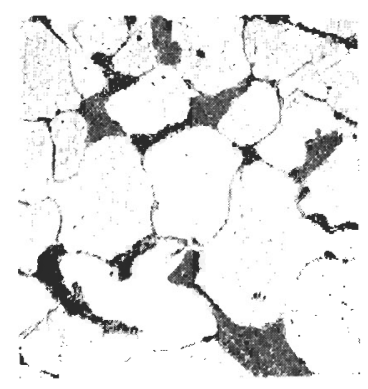

d

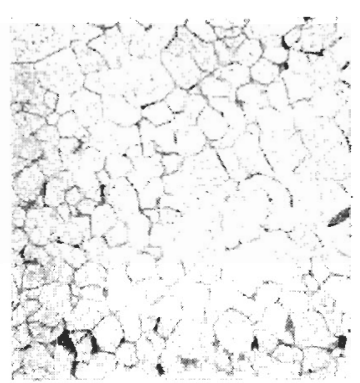

c

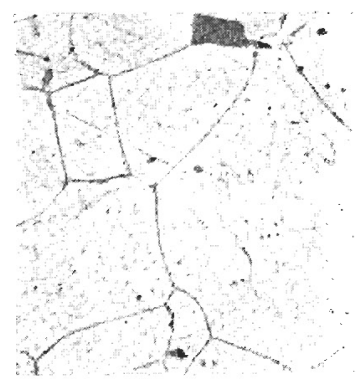

e

Авв. 7.-Technologisches Schema des Blattes, welches beiderseits vom Mittelgrat eine Zwischenlage mit gezacktem Rand und einen schmalen Streifen hat (a), und die Mikrostruktur des Blattes (Vergr. 100 X): b-c) Kasdanga; d-e) Durbe. 
Vergleichen wir das Metall der Blätter der Lanzenspitzen aus Kasdanga und Durbe mit einander, so sehen wir, daß beide Blätter aus Metallgleicher Zusammensetzung hergestellt sind. Ausnahme bildet der Mittelteil, in dem das erste Blatt bis $0,1 \%$ enthält- und das zweite Blatt 0,1$0,2 \%$ Kohlenstoff enthält. Außerdem hat die Lanzenspitze aus Durbe gröbere, natürliche Körner. An den Stellen der Zwischenlagen mit gezacktem Rand und Streifen, findet man verhältnismäßig grobkörnigen Ferrit, der sich schlecht ätzen läßt. Seine Mikrostruktur ist annähernd gleich der, auf der Abb. 10-c gezeigten Struktur.

Auf der Abb. 8 ist die Makrostruktur zweier Lanzenspitzen gezeigt: a) der Lanzenspitze aus Iwande nach dem Ätzen mit Reagens von Heine (10-12\% $\mathrm{CuCl}_{2} \mathrm{NH}_{4} \mathrm{Cl}$ Wasserlösung); b) der Lanzenspitze aus Durbe (Abb. 5-c) nach dem Ätzen mit Reagens von Heine, und c) derselben Lanzenspitzen aus Durbe nach dem Ätzen mit dem Klemmschen Reagens zur Bestimmung des Phosphorgehalts.

Das Reagens von Heine verleiht eine dunkle Ätzung an den Stellen des Eisens, 'wo daß Eisen mehr Beimischungen, wie Kohlenstoff, Schwefel und Phosphor, hat. Solche Stellen sind die Streifen und die Zwischenlagen mit gezacktem Rand. Jedoch, wie man bereits oben, bei der Analyse der Mikrostruktur, hatte sehen können, enthält das Metall der Streifen und Zwischenlagen mit gezacktem Rand keinen Kohlenstoff. Daneben, bei der Prüfung des Schwefelabdruckes (nach der Methode von Baumann ${ }^{7}$ ) konnte man feststellen, daß das Metall auch keine fühlbaren

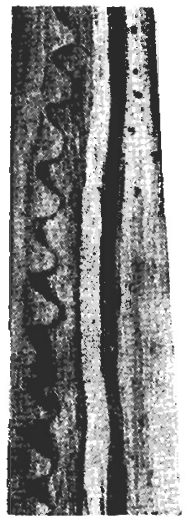

a

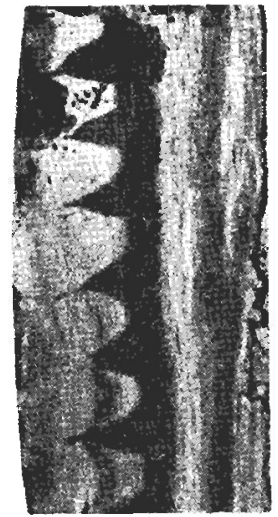

b

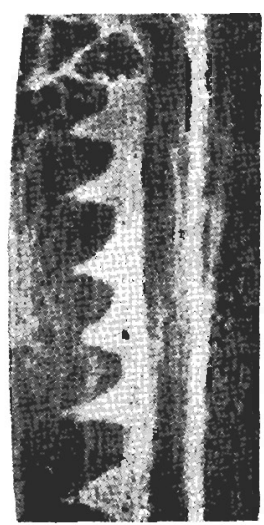

c

Aвв. 8.-Die Makrostruktur des Blattes: a) Iwande (nat. Gr.); b-c) Durbe (Vergr. 1, 6 X). 
Mengen des Schwefels enthält. Nachdem, beim Ätzen der Oberfläche des Blattes der Lanzenspitze aus Durbe mit Klemmschen Reagens, welches die Möglichkeit gibt, die Anwesenheit des Phosphors zu bestimmen, erhielt man das auf der Abb. 8-c gezeigte Bild. Darauf sieht man, daß der Streifen und die Zivischenlage mit gezacktem Rand hell geworden sind, während der übrige Teil der Oberfläche dunkel geblieben ist $\mathrm{Da}$ das Reagens von Klemm die Stellen, die eine größere Phosphormenge erhalten, hell bleiben läßt, so ist aus der Analyse zu folgen, daß das Metall der Streifen und Zwischenlagen eine größere Phosphormenge enthält. Von dem größeren Phosphorgehalt in den Schichten der Streifen und Zwischenlagen mit gezacktem Rand zeugt auch die Tatsache, daß in der Mikrostruktur dieser Stellen, grobkörniger Ferrit zu sehen ist, da die Anwesenheit des Phosphors die Grobkörnigkeit begünstigt.

Solche Phosphorgehaltproben wurden auch mit den Blättern der Lanzenspitzen aus Kasdanga und Aiskraukle unternommen, wobei das Ergebnis das Gleiche war, wie bei der Lanzenspitze aus Durbe.

Ebenso wie mit den vorhererwähnten Lanzenspitzen, kann man auch die Lanzenspitzen dieser Gruppe in Untergruppen einteilen. So ist z.B. die Lanzenspitze mit einer Zwischenlage mit gezacktem Rand aus Alsunga (Abb. 5-a) den Lanzenspitzen aus Kapenieki und Haimre ähnlich, aber die Lanzenspitze aus See Wilkmuischas (Abb. 5-b) ist einer Lanzenspitze aus Durbe (LM 13192) gleich. Ähnliche Lage ist auch bei den Lanzenspitzen, die einen Streifen und eine Zwischenlage mit gezacktem Rand haben: die Lanzenspitzen aus Durbe und Iwande (Abb. 5-c und d) sind denen aus Randwere, Kalsnawa, Estland, Piebalga, Salaspils und Schaureiti ähnlich; die Lanzenspitze aus Kasdanga (Abb. 5-e) sind drei aus Durbe, zwei aus Aiskraukle und Allaschi, einer aus Medse, Pasilciems, Bukaischi, Tukums, Aispute, Rauna, Haljala und einer aus See Wilkmuischas (LVM 1763) ähnlich.

\section{Die Lanzenspitzen mit komplizierter Damaszierung}

Dieser Gruppe hätte man 5 Lanzenspitzen zuzählen: ein Exemplar mit Zwischenlage (mit gezacktem Rand) und zwei schmalen Streifen (Durbe, LM 13215); ein mit gedrehter Zwischenlage (Ukmerge-UM) und drei mit Zwischenlage (mit gezacktem Rand) und gedrehter Zwischenlage (Durbe, LM 13115; Tarlia, PM 1/A1:25, und Saldus, LVM-V 8268:4). Drei von diesen Lanzenspitzen sind auf der Abb. 9 zu sehen.

Die längste von diesen Lanzenspitzen ist eine aus Durbe $(\mathrm{L}=$ $347 \mathrm{~mm}$ ), die kürzeste — die aus Tarlia ( $\mathrm{L}=$ etwa $290 \mathrm{~mm}$ ). Die 
kürzeste Tülle hat die Lanzenspitze aus Ukmerge $(\mathrm{L}=78 \mathrm{~mm})$ und die längste Tülle — die aus Saldus ( $\mathrm{L}=$ annähernd $110 \mathrm{~mm}$ ).

Die Querschnitte wurden bei beiden Lanzenspitzen aus Durbe er-

AвB. 9.-Die Lanzenspitzen mit komplizicrter Damaszierung: a) Durbe (LM 13215; 1/3); b) Ukmerge (1/3); c) Dumbe (LM 13115; 1/3).-Die Sielle des Querschnitis.

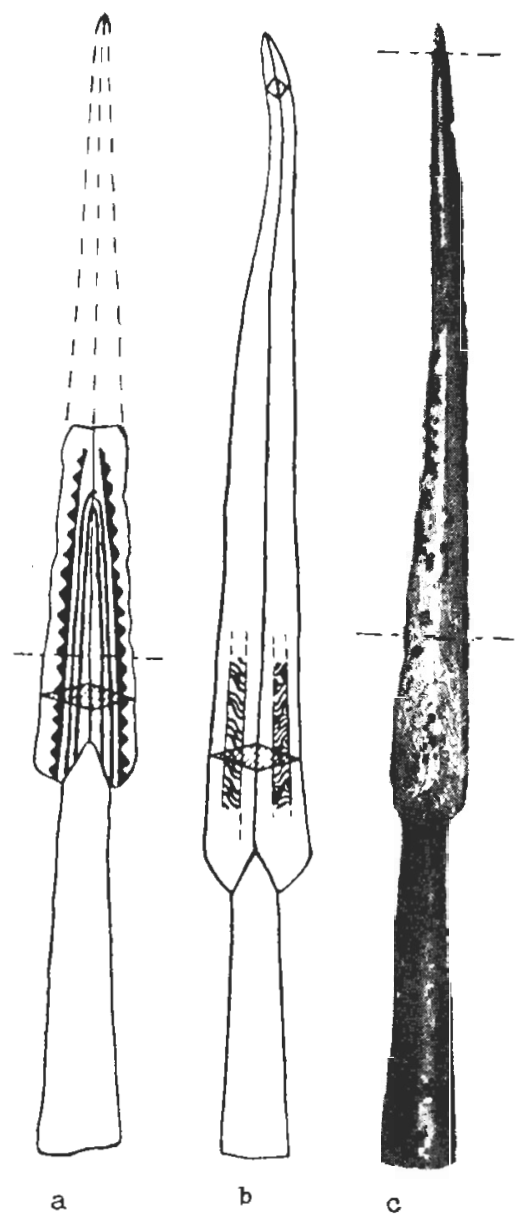




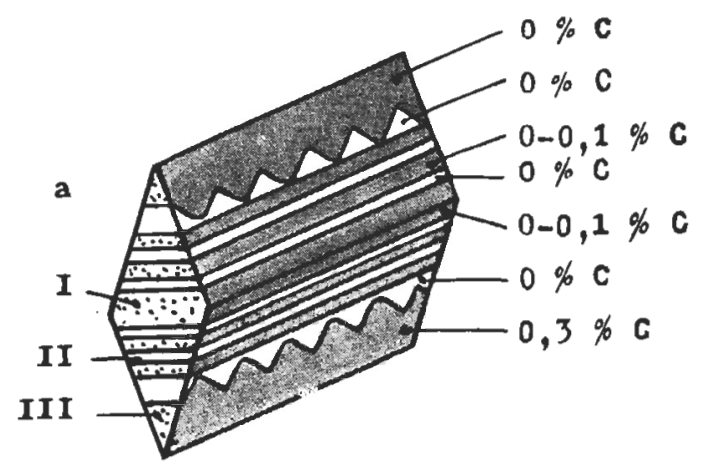

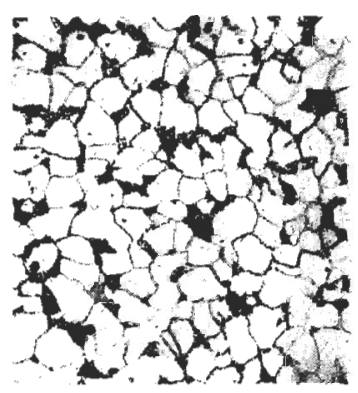

b

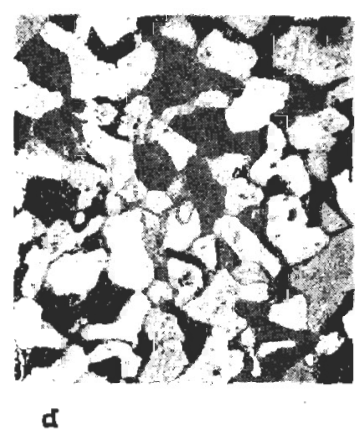

Авв. 10.-Technologisches Schema des Blattes, welches beiderseits vom Millelgrat eine Zwischenlage mit gezacktem Rand und zwei scbmalen Streifen hat (a) und die Mikrostruktur (Vergr. 100 X) des Durbe. Blattes ( $b$ und $c$ ). 


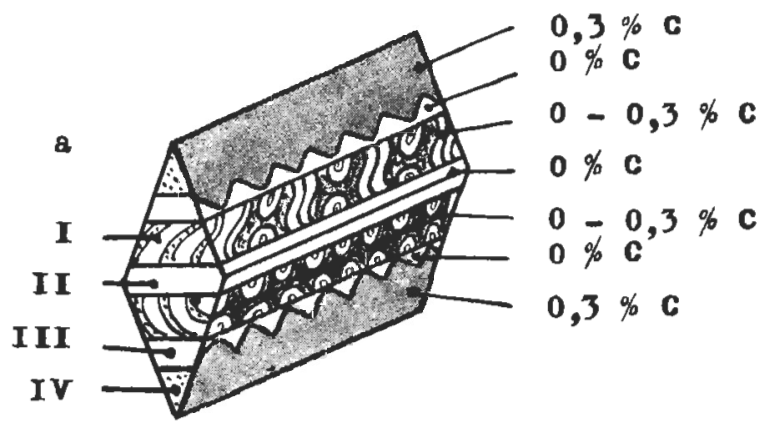

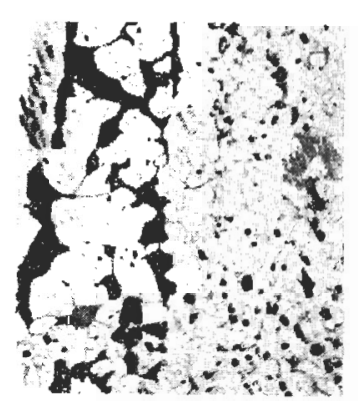

b

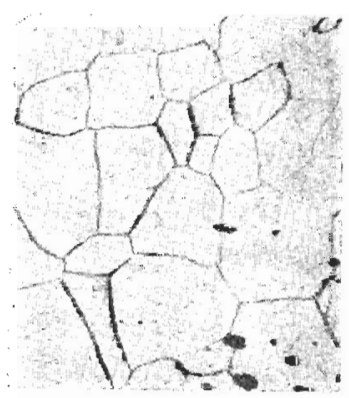

d

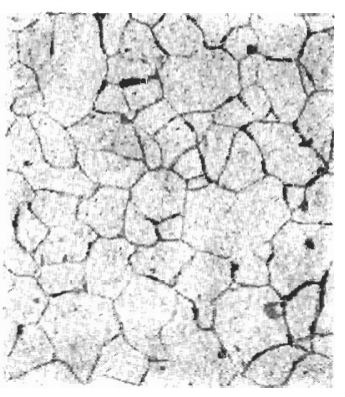

c

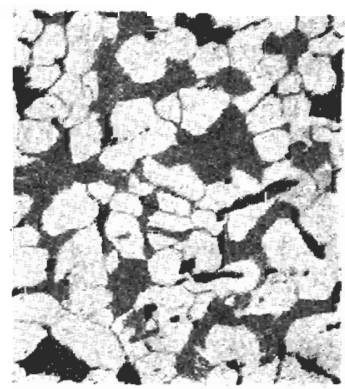

e

Авв. 11.-Tecbnologiscbes Scbema des Blattes, welches beiderseits vom Mittelgrat eine gedrebte Zwischenlage und Zwischenlage mit gezacktem Rand bat (a) und die Mikrostruktur (Vergr. $100 \mathrm{X}$ ) des DurbeBlattes $(b-e)$. 
forscht. Das technologische Schema (Abb. 10-a) und die Mikrostruktur (Abb. 10-bd) der ersten, das technologische Schema (Abb. 11-a) und die Mikrostruktur (Abb. 11-be) der zweiten, sind auf den, im Klammern angegebenen Abbildungen zu sehen (Abb. 10, 11 und 12).

Aus dem technologischen Schema (Abb. 10-a) des Blattes mit einer Zwischenlage mit gezacktem Rand und zwei schmalen Streifen aus Durbe geht hervor, daß das Metall einzelner Schichte denselben Kohlenstoffgehalt aufweist, wie das einstreifige Blatt aus Kaleti (Abb. 3-a) und das Blatt mit einer Zwischenlage mit gezacktem Rand aus Alsunga (Abb. 6-a).

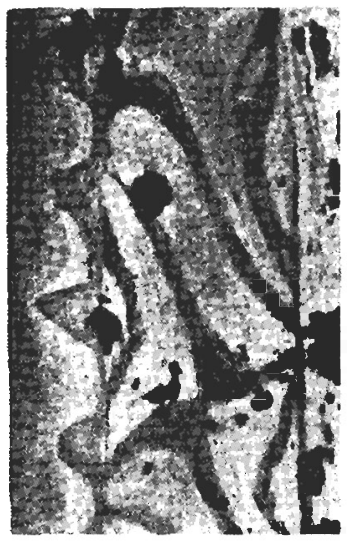

a

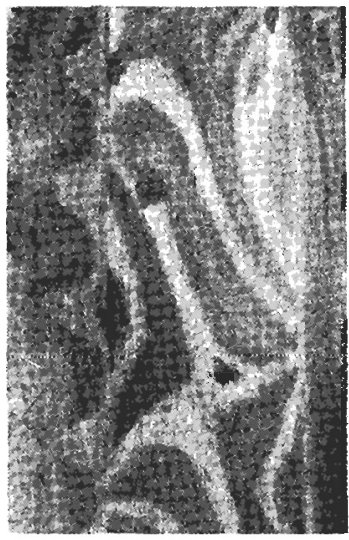

Aвв, 12.-Die Makrostruktur des Durbe-Blattes (Vergr. $3 X)$.

Der Unterschied besteht nur darin, daß hier, wie auch bei dem einstreifigen Blatt aus Durbe (Abb. 3-a), die eine Schneidenspitze aus Eisen (0\% C) geschmiedet ist. Die Mikrostruktur des Mittelteiles - Stelle I (Abb. 10-a) ist auf der Abb. 10-b zu sehen (man sieht Ferrit und Perlit), die Mikrostruktur des Streifens (Stelle II) ist auf der Abb. 10-c gezeigt (man sieht einen groben Ferritkern), und die der Schneidenspitze (Stelle III) ist auf der Abb. 10-d zu sehen (man sieht den Ferrit und Perlit). Beim Stählen konnte man nur die eine Schneidenspitze härter erhalten.

Das technologische Schema des Blattes mit einer Zwischenlage mit gezacktem Rand und einer gedrehten Zwischenlage der Lanzenspitze aus Durbe ist auf der Abb. 11-a zu sehen. Man sieht, daß der Mittelteil des Blattes aus Eisen ist (die Mikrostruktur der Stelle II ist auf der Abb. 11-c gezeigt, wo man kleine Ferritkörner erblicken kann); die gedrehte Zwischenlage besteht aus Eisen in dem, vor der Zusammendrehung, Stahlla- 
mellen mit Kohlenstoffgehalt bis $0,3 \%$ eingeschweißt sind (ihre Mikrostruktur ist auf der Abb. 11-b gezeigt, 'wo in der linken Hälfte ein FerritPerlitstreifen ist, in der rechten Hälfte - Ferrit mit feinen Schlackeneinschließungen zu sehen ist); die Zwischenlage mit gezacktem Rand besteht aus Eisen (auf der Abb. 11-d ist Ferrit der Stelle III gezeigt man sieht verhältnismäßig grobe Körner) und die Schneidenspitze ist aus Stahl mit Kohlenstoffgehalt 0,3\% (auf der Abb. 11-d ist die Struktur der Stelle IV gezeigt, welche aus Ferrit und Perlit besteht). Wie man die gedrehte Zwischenlage herstellt, ist in unseren früheren Arbeiten berichtet ${ }^{2,3}$. Es ist zu vermerken, daß die Schneide-Eigenschaften dieser Lanzenspitze geringer sind, als die der Lanzenspitzen mit gleicher Damaszierung, die eine versilberte Tülle oder breite Blätter mit langen Tüllen haben, weil ihre Schneidenspitzen 0,8-0,9\% Kohlenstoff enthalten ${ }^{2,3}$.

Analyse der Phosphormenge zeugte von einem erhöhten Phosphorgehalt in der Zwischenlage mit gezacktem Rand (siehe Abb. 12-a, auf der man dunkle Zacken dieser Schicht nach dem Ätzen mit Heines Reagens sehen kann; und Abb. 12-b zeigt dieselbe Schicht nach dem Ätzen mit Klemmschen Reagens), aber in der gedrehten Zwischenlage erhöhte Phosphormenge enthalten die Stahllamellen (siehe Abb. 12-a, auf der man nach dem Ätzen mit Heines Reagens dunkle Streifen des Stahls dieser Schicht sehen kann und auf der Abb. 12-b dieselben in heller Farbe nach dem Ätzen mit Klemmschen Reagens).

\section{Spektralanalyse}

Um eine Übersicht zu gewinnen, welche legierenden Elemente die analysierten Lanzenspitzen enthalten (was das Herstellungszentrum zu ermitteln helfen 'würde), wurde eine Spektralanalyse unternommen, welche die Ermittlung von Nickel, Molybdän, Vanadium, Kobalt, Titan, Chrom und Wolfram zum Ziele hatten. Bei der Spektralanalyse der Blätter von 26 Lanzenspitzen wurde festgestellt, daß 12 Exemplare kein von oben erwähnten Elementen enthalten, 8 Exemplare enthalten Spuren von Nickel (bis $0,05 \%$ ), 5 Exemplare enthalten $0,1-0,15 \%$ und ein Exemplar - 0,16-0,26\% Nickel (II Tabelle).

Es ist aber gleich zu vermerken, daß nicht alle Stellen des Blattes die gleiche Menge Nickel enthalten. So gab z.B. die Analyse einer Lanzenspitze aus Aiskraukle (LVM-I 3153 ) folgende Resultate: 0,16\%, 0,19\% und $0,26 \%$; der zweiten Lanzenspitze aus Aiskraukle (LVM-I 3108): $0,11 \%, 0,13 \%$ und $0,40 \%$; einer Lanzenspitze aus Durbe (LM 13215): $0,05 \%$ und $0,13 \%$; der zweiten Lanzenspitze aus Durbe (LM 13230): 
$0,05 \%$ und $0,10 \%$. Solche ungleichmäßige Nickelverteilung erschwert die Ausgabe des Ermittlung des Herstellungszentrum der Lanzenspitzen.

\section{Zusammenfassung}

Bei der metallographischen Analyse von 65 kurischen rhombischen Lanzenspitzen, waren 7 Arten der Damaszierung zu unterscheiden: 1) das Blatt hat beiderseits vom Mittelgrat einen schmalen Streifen (15 Ex.); 2) das Blatt hat beiderseits vom Mittelgrat zwei schmale Streifen ( 8 Ex.); 3) das Blatt hat beiderseits vom Mittelgrat eine Zwischenlage mit gezacktem Rand ( 6 Ex.); 4) das Blatt hat beiderseits vom Mittelgrat eine Zwischenlage mit gezacktem Rand und einen schmalen Streifen (31 Ex.); 5) das Blatt hat beiderseits vom Mittelgrat eine Zwischenlage mit gezacktem Rand und zwei schmale Streifen (1 Ex.); 6) das Blatt hat beiderseits vom Mittelgrat eine gedrehte Zwischenlage (1 Ex.), und 7) das Blatt hat beiderseits vom Mittelgrat eine gedrehte Zwischenlage und eine Zwischenlage mit gezacktem Rand ( 3 Ex.).

40 von den analysierten Lanzenspitzen wurden auf dem von den kurischen Stämmen bewohnten Gebiet gefunden, die übrigen 25 Exemplarc - auf breitem ihm anliegenden Territorium (Abb. 1).

Die Ornamentierung wurde durch geschickte Schmiedeschweißarbeit erreicht, indem man Eisen mit phosphorenthaltendem Eisen oder mit verschieden zusammengesetztem Stahl zusammenschweißte. Ein auf diese Weise hergestelltes Blatt hat eine höhere Elastizitätsgrenze im Vergleich mit einem Blatt aus gewöhnlichem Eisen oder mit einem Blatt aus Stahl mit geringerem Kohlenstoffgehalt; es sieht auch dazu schöner aus.

Man muß aber bemerken, daß die Schneidenspitzen dieser Lanzen mit geringerer Schneidefähigkeit sind als die besten Lanzenspitzen des XI Jh.'s mit einer versilberten Tülle, und als viele Lanzenspitzen des XII.-XIII. Jh.'s mit breiten Blättern und langen Tüllen, welche einen richtigeren Kohlenstoffgehalt in den Schneidenspitzen haben. Und sie ermöglichen dadurch nach dem Härten, bessere Schneideeigenschaften $\mathrm{zu}$ verleihen. 


\section{TABELLE I}

\begin{tabular}{|c|c|c|c|c|c|}
\hline \multirow[b]{2}{*}{ NR. } & \multirow[b]{2}{*}{ GRUPPE } & \multirow[b]{2}{*}{ DAMASZIERUNG DES BLATTES } & \multirow[b]{2}{*}{ STÜCK } & \multicolumn{2}{|c|}{ STÜCK DER ANALYSE } \\
\hline & & & & $\begin{array}{l}\text { DER MIKRO- } \\
\text { STRUKTUR }\end{array}$ & $\begin{array}{l}\text { DER PHOS- } \\
\text { PHORMENGE }\end{array}$ \\
\hline 1 & $\mathrm{I}$ & Ein schmaler Streifen ........... & 15 & 3 & 1 \\
\hline 2 & II & Zwei schmale Streifen ........... & 8 & 2 & 1 \\
\hline 3 & IV & $\begin{array}{l}\text { Zwischenlagen mit gezacktem } \\
\text { Rand }\end{array}$ & 6 & 1 & - \\
\hline 4 & $\mathrm{~V}$ & $\begin{array}{l}\text { Zwischenlagen mit gezacktem } \\
\text { Rand und einem schmalen } \\
\text { Streifen } \ldots \ldots \ldots \ldots \ldots \ldots \ldots \ldots\end{array}$ & 31 & 3 & 3 \\
\hline 5 & VI & $\begin{array}{c}\text { Zwischenlagen mit gezacktem } \\
\text { Rand und zwei schmalen } \\
\text { Streifen ...................... }\end{array}$ & 1 & 1 & - \\
\hline 6 & VIII & Eine gedrehte Zwischenlage ... & 1 & - & - \\
\hline 7 & XI & $\begin{array}{l}\text { Eine gedrehte Zwischenlage } \\
\text { und Zwischenlage mit ge- } \\
\text { zacktem Rand ................. }\end{array}$ & 3 & 1 & 1 \\
\hline & & ZUSAMMEN ......... & 65 & 11 & 6 \\
\hline
\end{tabular}

\section{TABELLE II}

\begin{tabular}{|c|c|c|c|c|c|}
\hline \multirow[b]{2}{*}{ GRUPPE } & \multirow[b]{2}{*}{ DAMASZIERUNG DES BLATTES } & \multicolumn{4}{|c|}{ NICKEL GEHALT, \% } \\
\hline & & $\begin{array}{l}\text { KEIN } \\
\text { NICKEL }\end{array}$ & $\begin{array}{l}\text { SPUREN VON } \\
\text { NICKEL } \\
\left(\text { BIS } 0,05^{\circ} \%\right)\end{array}$ & $\begin{array}{c}0,1-0,15 \% \\
\text { NICKEL }\end{array}$ & $\begin{array}{c}0,16-0,26 \% \\
\text { NICKEL }\end{array}$ \\
\hline I & Ein schmaler Streifen ..... & 4 & - & - & - \\
\hline II & Zwei schmale Streifen ..... & 1 & 2 & - & - \\
\hline IV & $\begin{array}{l}\text { Zwischenlagen mit gezack- } \\
\text { tem Rand } . . . \ldots \ldots \ldots \ldots \ldots \ldots . . .\end{array}$ & 2 & 2 & - & 1 \\
\hline $\mathrm{V}$ & $\begin{array}{l}\text { Zwischenlagen mit gezack- } \\
\text { tem Rand und einem } \\
\text { schmalen Streifen ....... }\end{array}$ & 5 & 3 & 4 & 1 \\
\hline VI & $\begin{array}{l}\text { Zwischenlagen mit gezack- } \\
\text { tem Rand und zwei }\end{array}$ & & & & \\
\hline \multirow{3}{*}{$X I$} & schmalen Streifen ...... & - & - & 1 & - \\
\hline & $\begin{array}{l}\text { Eine gedrehte Zwischenla- } \\
\text { ge und Zwischenlage mit } \\
\text { gezacktem Rand ......... }\end{array}$ & - & 1 & - & - \\
\hline & ZUSAMMEN .......... & 12 & 8 & 5 & 1 \\
\hline
\end{tabular}




\section{LITERATURVERZEICHNIS}

1. A. AnTEINs: Dzelzs un tērauda izstrādäjumu struktūras, ipašìnas un izgatavošanas tebnoloğija senajā Latvijā: «Arheoloğija un etnografija», II, Rìga, 1960.

2. A. Anteins: Senās Latvijas damascètā tērauda škēpu smaili: «Arheologija un etnografija», IV, Rīga, 1962.

3. A. ANTEINS: Nakonetschniki kopij iz swarotscbnoj uzortsbatoj (damasskoj) siali $w$ drewnej Pribaltike: «Sowetskaja archeologija», Nr. 4, 1963, Moskva.

4. A. Anterns: Nakonetscbniki kopij iz swarotscbnoj uzortschatoj (damasskoj) stali, naidennije $w$ Estonii: «Izvestija AN Est. SSR», ser. XI, 4, 1964, Tallin.

5. A. Anteins: Drewneje oruscbije iz damasskoj stali $w$ Litwe: «Materialy VI konferenzii po istorii nauki w Pribaltike», Vilnjus, 1965.

6. A. Anteıns: Im Ostbalikum gefundene Scbwerter mit damaszierten Klingen: «Waffen- und Kostümkunde», Nr. 2, 1966, München-Berlin.

7. M. BECKERT und H. KLEM : Handbuch der metallograpbischen Ätzverfabren, Leipzig, 1962.

8. Latvijas senatne. Celvedis pa Latvijas PSR Vèstures muzeju, Rìga, 1959.

9. A. LiestöL:: Blodrefill og mil: «Wiking», XIV, Oslo, 1951.

10. P. Stepinš: Durbes kauja 1260.g., Rīga, 1967. 